Biotempo (Lima)

ORIGINAL ARTICLE / ARTÍCULO ORIGINAL

DESCRIPTION OF THE INTRAOCULAR PRESSURE (IOP) IN RABBITS (ORYCTOLAGUS CUNICULUS (LINNAEUS, 1758)) SUBMITTED TO TWO ANESTHETIC PROTOCOLS

\title{
DESCRIPCIÓN DE LA PRESIÓN INTRAOCULAR (PIO) EN CONEJOS (ORYCTOLAGUS CUNICULUS (LINNAEUS, 1758)) SOMETIDOS A DOS PROTOCOLOS ANESTÉSICOS
}

\author{
Luz Diana Mori-Malaver ${ }^{1}$; Verónica Alvarez-Begazo de Jara ${ }^{1}$ \& Mauricio Jara-Aguirre ${ }^{1}$
}

1 Laboratorio de Cirugía (LA82). Escuela profesional de Medicina Veterinaria. Facultad de Ciencias Biológicas. Universidad Ricardo Palma. Av. Benavides 5440, Lima 33, Perú.

Author for correspondence: Ldmm1991@gm .-mjara@urp.edu.pe

\begin{abstract}
The main objective of this work was to describe intraocular pressure (IOP) in rabbits (Oryctolagus cuniculus (Linnaeus, 1758)) subjected to two anesthetic protocols. Anesthetic protocols 1 and 2 were Meloxicam-Xylazine-Ketamine and Meloxicam-Diazepam-Ketamine, respectively. It was a cross-sectional study of descriptive nature only, with a sample of twelve rabbits divided into two groups for the use of anesthetic protocols 1 and 2 independently. The IOP was measured with a Shiotz tonometer throughout the duration of the anesthetic protocols at minutes 5, 10 and 15. Subsequently, based on the measurements collected, the statistical frequencies were determined and the results obtained show the reduction of significant IOP during anesthesia in both anesthetic protocols, especially at minute 10 with measurements of 9.017 and $9.442 \mathrm{mmHg}$ considering the baseline measurements of 19.917 and $20.408 \mathrm{mmHg}$ and a normal IOP value in rabbits between 15 and $23 \mathrm{mmHg}$. Using anesthetic protocols composed of drugs that reduce or help keep IOP low, such as Xylazine and Diazepam, used in daily practice with ketamine as an inductor drug. It is important to consider the IOP when formulating anesthetic protocols in rabbits, since a considerable increase in IOP could mean the occurrence of ocular damage or the exacerbation of some ocular pathology prior to anesthesia. The results of this study provide a contribution to consider when establishing anesthetic protocols in rabbits and represent important data for the formulation of new research work in the same area of study.
\end{abstract}

Key words: Intraocular pressure - rabbits - xylazine

\section{RESUMEN}

El presente trabajo tuvo como objetivo principal la descripción de la presión intraocular (PIO) en conejos (Orcytolagus cuniculus (Linnaeus, 1758)) sometidos a dos protocolos anestésicos. Los protocolos anestésicos 1 y 2 fueron de Meloxicam-Xilacina-Ketamina y Meloxicam-Diazepam-Ketamina respectivamente. Se trató de un estudio transversal de 
índole descriptivo únicamente, con una muestra de doce conejos divididos en dos grupos para el uso de los protocolos anestésicos 1 y 2 independientemente. Se realizó la medición de la PIO con un tonómetro de Shiotz a lo largo de la duración del cuadro anestésico en los minutos 5, 10 y 15. Posteriormente y a partir de las mediciones recabadas se determinaron las frecuencias estadísticas y los resultados obtenidos demuestran la reducción de la PIO significativa durante anestesia en ambos protocolos anestésicos, de manera especial al minuto 10 con mediciones de 9.017 y 9.442 $\mathrm{mmHg}$ considerando las medidas basales de 19.917 y $20.408 \mathrm{mmHg}$ y un valor normal de PIO en conejos de entre 15 a $23 \mathrm{mmHg}$. El empleo de protocolos anestésicos compuestos por fármacos que disminuyen o ayudan a mantener baja la PIO como la Xilacina y el Diazepam, son utilizados en la práctica diaria con la ketamina como fármaco inductorio. Resulta importante considerar la PIO al momento de formular protocolos anestésicos en conejos, ya que un alza considerable en PIO podría significar la ocurrencia de un dańo ocular o la exacerbación de alguna patología ocular previa a anestesia. Los resultados de este estudio significan un aporte a considerar al momento de establecer protocolos anestésicos en conejos y representan datos importantes para la formulación de nuevos trabajos de investigación en la misma área de estudio.

Palabras clave: Conejos - presión intraocular - xilacina

\section{INTRODUCCIÓN}

Como animales de compañía, los conejos han pasado a ser pacientes con un creciente número de ingresos a centros veterinarios en los últimos ańos, tal y como lo han sido los perros durante mucho tiempo (Zamira et al., 2014). Asociado a este incremento en la atención veterinaria y el uso de conejos como modelos experimentales por su fácil manejo y manutención (Hedenqvist, 2005), numerosos estudios se han venido desarrollando en cuanto a su salud general y enfermedades de índole ocular (Kern, 1997).

Fármacos pre-anestésicos y anestésicos son minuciosamente seleccionados teniendo en cuenta el estado de salud del animal y el motivo por el cual éste necesite ser anestesiado, como un manejo clínico de inspección o un procedimiento quirúrgico (Kern, 1997). Uno de los fármacos de mayor uso en protocolos anestésicos, es la Ketamina, la cual actúa eficazmente como anestésico inductorio; sin embargo, puede ocasionar un aumento temporal de la presión intraocular (PIO) (Ghaffari \& Moghaddassi, 2010). Se considera al aumento de la PIO como un factor predisponente y causa principal de patologías oculares, como el glaucoma (Pietro-Calvo, 2012). Por otro lado el uso de fármacos pre-anestésicos tienden a disminuir la PIO (Raw \& Mostafa, 2001).

El presente trabajo tiene como fin el describir la presión intraocular (PIO) de conejos, Oryctolagus cuniculus (Linnaeus 1758), sometidos a dos protocolos anestésicos: Protocolo 1 (Meloxicam, Xilacina y Ketamina) y Protocolo 2 (Meloxicam, Diazepam y Ketamina), para observar las variaciones de PIO que se puedan obtener.
La información resultante de las mediciones de PIO, al final de este proyecto servirá de apoyo para el Médico Veterinario y a la realización de mejores protocolos anestésicos en conejos.

\section{MATERIALES Y MÉTODOS}

Tipo y diseño de estudio

Se trata de un estudio descriptivo y transversal de la PIO.

Variables

Las variables consideradas en este estudio están relacionadas a los fármacos utilizados dentro de cada protocolo establecido y la influencia de los mismos en la PIO. Se mencionan a continuación para luego ser definidas en la operacionalización de las mismas:

- PIO al usar Protocolo anestésico 1 (Meloxicam, Xilacina, Ketamina)

- PIO al usar Protocolo anestésico 2 (Meloxicam, Diazepam, Ketamina).

Muestra

Los conejos fueron seleccionados de las clases de "Anestesiología Veterinaria y preoperatoria" e "Instrumentación Quirúrgica y Anestesiología" del ciclo 2019-1. Ambos cursos de la carrera de Medicina Veterinaria en la Universidad Ricardo Palma (URP), Lima, Perú. 
Para la determinación del tamaño muestral de conejos necesarios para este trabajo de investigación, se utilizó la fórmula de "Tamaño de la muestra para una población finita", de donde se obtuvo un tamaño muestral de 12 conejos necesarios para realizar este estudio.

Procedimiento (Castellvi et al., 2009; Snyder et al., 2018)

- Se necesitó al experimentador y un auxiliar para una correcta sujeción del animal. Ambos llevaron guantes de látex, utilizados para exámenes de rutina.

- Se colocó 1 gota en cada ojo de Proparacaína (anestésico tópico oftálmico) y se debió esperar 5 min para llegar al efecto deseado del anestésico.

- Pasados los 5 min, se colocó al animal en decúbito lateral derecho, para proceder a realizar la medición del ojo izquierdo (En decúbito lateral izquierdo para la medición del ojo derecho).

- Delicadamente, se posicionó la cabeza del animal, de manera tal, que la córnea del ojo estuvo centrada.

- Con ayuda manual, se mantuvieron los párpados abiertos e inmediatamente se ubicó el tonómetro de Schiotz encima y en contacto directo con la córnea; quedando de manera perpendicular a la misma y se esperó a que la aguja del tonómetro de una medida.

- La medición con el tonómetro de Schiotz se realizó con pequeńas pesas de 5,5, 7,5 y 10 $\mathrm{g}$ independientemente, por lo cual se hizo la medición descrita en el paso $\mathrm{n}^{\circ} 5$, tres veces por cada ojo de cada conejo involucrado en este estudio. Asimismo, se calibró a "cero" dicho instrumento, entre cada medición.

- Se desinfectó el pabellón del cilindro y la parte distal del vástago movible que soporta el peso del tonómetro de Schiotz (partes del tonómetro de Schiotz que entran en contacto directo con el ojo del animal), entre medición de cada ojo y por supuesto entre medición de cada animal. La desinfección que se realizó con algodón embebido en alcohol etílico de $96^{\circ}$ y posteriormente se pasó un algodón embebido en suero fisiológico; para evitar irritaciones oculares indeseadas, debido al alcohol.

- La medición de la presión intraocular, como tal, tomó alrededor de $3-5$ min como máximo en cada conejo.

- Como profiláctico y pasado el efecto anestésico, se administró una gota de antibiótico tópico en cada ojo de cada animal.

Una vez obtenidas las medidas basales, se procedió a tomar las mediciones de la PIO en conejos sometidos a los dos protocolos anestésicos establecidos en este estudio. Por lo cual, de manera aleatoria se dividieron los 12 conejos, en grupos de 6 cada uno. Los protocolos anestésicos usados son descritos a continuación (Tabla 1):

Tabla 1. Protocolos anestésicos usados en las mediciones de la PIO (Presión Intraocular) en conejos.

\begin{tabular}{|c|c|c|c|c|c|c|}
\hline & \multicolumn{3}{|c|}{$\begin{array}{c}\text { Grupo A } \\
\text { Protocolo anestésico } 1\end{array}$} & \multicolumn{3}{|c|}{$\begin{array}{c}\text { Grupo I } \\
\text { Protocolo anestésico } 2\end{array}$} \\
\hline & Fármacos & Dosis* (mg/kg) & $\begin{array}{l}\text { Dosis del } \\
\text { presente } \\
\text { estudio (mg/ } \\
\mathrm{kg} \text { ) }\end{array}$ & Fármacos & $\begin{array}{l}\text { Dosis* (mg/ } \\
\mathrm{kg})\end{array}$ & $\begin{array}{l}\text { Dosis del } \\
\text { presente } \\
\text { estudio }(\mathrm{mg} / \\
\mathrm{kg})\end{array}$ \\
\hline & Meloxicam & 0,3 (SC) & $0,3(\mathrm{SC})$ & Meloxicam & 0,3 (SC) & $0,3(\mathrm{SC})$ \\
\hline Pre - anestésico & Xilacina & 5 (IM) & $2,5(\mathrm{IM})$ & Diazepam & $1-5$ (IM) & 2 (IM) \\
\hline Anestésico & Ketamina & 25-35 (IM) & 25 (IM) & Ketamina & 20-40 (IM) & 20 (IM) \\
\hline
\end{tabular}

*Las dosis fueron obtenidas de UC (2016). 
Una vez tomadas las mediciones basales se procedió a tomar la PIO utilizando los protocolos anestésicos 1 y 2.

El procedimiento para completar dicha tarea fue el mismo descrito anteriormente para la toma de mediciones basales de PIO. Acotando que en el paso $n^{\circ} 6$ se midió la PIO a los 5, 15 y 20 min durante anestesia, en cada ojo de cada conejo y que en el paso $\mathrm{n}^{\circ} 9$, se aplicó el profiláctico una vez que el conejo estuviere recuperado del cuadro anestésico.

Todas las mediciones obtenidas fueron convertidas con la tabla de Umrechnungstabelle de 1955 a valores en mmHg presentada a continuación, de las cuales se calculó una media para hallar el valor final de la PIO de cada ojo en cada conejo.

Los datos obtenidos de las medidas basales y durante ambos protocolos anestésicos fueron traspuestos en el programa estadístico, IBM ${ }^{\circledast}$ SPSS $^{\circledast}$ Statistics, de donde se determinaron frecuencias estadísticas descriptivas para su posterior análisis e interpretación.

\section{Aspectos éticos}

Antes de realizar el trabajo de campo de este estudio, fue necesaria la creación de un Consentimiento Informado, el cual se entregó a cada propietario de los 12 conejos requeridos. Este tuvo como propósito el informar sobre el trabajo a realizar con sus mascotas y todas las implicancias del mismo; además de obtener su aprobación para trabajar con sus animales.

\section{RESULTADOS}

La data recabada traspuesta en el programa de estadísticas, arrojó los siguientes resultados expuestos a continuación (Tabla 2):

Tabla 2. Frecuencias estadísticas en Protocolo anestésico 1 (Meloxicam, Xilacina y Ketamina).

\begin{tabular}{|c|c|c|c|c|}
\hline & $\begin{array}{l}\text { Mediciones basales } \\
\text { Grupo A }\end{array}$ & $\begin{array}{l}\text { Mediciones Grupo } \\
\text { A protocolo } \\
\text { anestésico } 1(\mathrm{~min} \\
\text { 5) }\end{array}$ & $\begin{array}{l}\text { Mediciones Grupo } \\
\text { A protocolo } \\
\text { anestésico } 1(\mathrm{~min} \\
10)\end{array}$ & $\begin{array}{l}\text { Mediciones Grupo A } \\
\text { protocolo anestésico } 1 \\
(\min 15)\end{array}$ \\
\hline Media & 19,91 & 15,51 & 9,01 & 8,35 \\
\hline Mediana & 17,85 & 15,90 & 10,90 & 8,60 \\
\hline Moda & 29,1 & 15,90 & $1,6^{*}$ & $0^{*}$ \\
\hline Desviación Estándar & 6,48 & 5,87 & 5,30 & 4,35 \\
\hline Varianza & 42,02 & 34,53 & 28,10 & 18,98 \\
\hline Asimetría & 0,41 & $-0,69$ & $-0,30$ & $-0,42$ \\
\hline $\begin{array}{l}\text { Error estándar de } \\
\text { asimetría }\end{array}$ & 0,63 & 0,63 & 0,63 & 0,63 \\
\hline Curtosis & $-1,49$ & $-0,36$ & $-1,48$ & $-0,43$ \\
\hline $\begin{array}{l}\text { Error estándar de } \\
\text { curtosis }\end{array}$ & 1,23 & 1,23 & 1,23 & 1,23 \\
\hline Mínimo & 12,3 & 4,0 & 1,6 & 0 \\
\hline Máximo & 29,1 & 22,1 & 15,8 & 15,0 \\
\hline \multirow[t]{3}{*}{ Percentiles } & 13,97 & 11,82 & 3,17 & 4,50 \\
\hline & 17,85 & 15,90 & 10,90 & 8,60 \\
\hline & 27,40 & 20,85 & 12,5 & 11,65 \\
\hline
\end{tabular}

(*) Se muestra valor más pequeño

Tal y como se muestra en la Tabla 2, se tomaron en cuenta un total de 12 mediciones de PIO, pertenecientes a los 6 conejos del Grupo A, considerando ambos ojos y sin pérdida de ningún dato. La media de las medidas basales es de 19,917 $\mathrm{mmHg}$, mientras que al utilizar el protocolo anestésico 1 (Meloxicam, Xilacina y Ketamina), se observa una media de $15,517 \mathrm{mmHg}$ al min 5 y luego disminuye a $9,017 \mathrm{mmHg}$ y $8,350 \mathrm{mmHg}$ al minuto 10 y 15 , respectivamente.
El valor de PIO más elevado obtenido fue de 22,1 $\mathrm{mmHg}$ al min 5, mientras que el mínimo valor es de 15,0 mmHg en la medición final al min 15. En cuanto a los valores obtenidos de desviación estándar y varianza, se observa una mayor variabilidad y dispersión de datos en las medidas basales, seguidas por las mediciones de PIO al min 5 y consecutivamente en menor grado al min $10 \mathrm{y}$ 15 . 
En cuanto a la forma de distribución de los datos en cuestión, se tiene una asimetría negativa, indicativo de una curva de distribución con cola orientada hacia la izquierda por poseer valores menores a la media con un error de asimetría del 0,66 indicando que no se trata de una distribución normal. Se obtuvo una curtosis negativa generando que dicha curva con asimetría negativa no sea tan alta, ya que los valores se encuentran más alejados de la media, debido a la variabilidad de valores ya mencionada y el error estándar de curtosis, el cual también rechaza la posibilidad de tratarse de una distribución normal.

Al tratarse de una distribución asimétrica y partir de los valores mínimos y máximos de $\mathrm{PIO}$, así como de los percentiles obtenidos, se realizó un gráfico Box-plot para los min 5, 10 y 15 (Fig. 1).

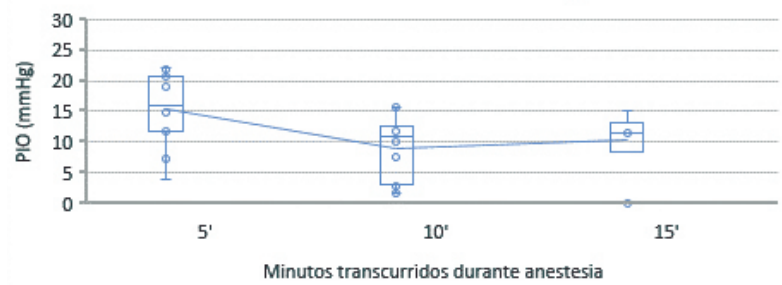

Figura 1. Distribución estadística de PIO - Protocolo anestésico 1 (Meloxicam, Xilacina y Ketamina).
La Fig. 1 se realizó sin tomar en cuenta las medidas basales, solo los valores de PIO utilizando el Protocolo anestésico 1 . Al min 5 se obtuvo un valor mínimo de PIO de 4,0 $\mathrm{mmHg}$, considerado como un valor por debajo del rango normal de PIO en conejos y uno máximo de 22,1 $\mathrm{mmHg}$, valores de mucha variabilidad por diferencias en el tiempo de acción de los fármacos en los especímenes evaluados.

El cuartil 1 (Q1) indica que el 25\% de los datos recabados se encuentran por debajo de 13,97 $\mathrm{mmHg}$ y el cuartil 3 (Q3) seńala que el 75\% de los datos se encuentran por encima de $20,85 \mathrm{mmHg}$; valores que se encontraron por debajo y dentro del rango normal PIO en conejos. Al min 10, se observa una reducción de todos los valores empezando los valores mínimo y máximo con 1,6 y 15,8 $\mathrm{mmHg}$ respectivamente, así como los demás valores encontrándose por debajo de los valores obtenidos al minuto 5. En el min 15; sin embargo, los valores se incrementan nuevamente, pero sin llegar a ser tan elevados como aquellos del min 5 . Además, se aprecia un valor atípico de 0,0 , debido a alguna variación en el efecto anestésico sobre el espécimen y máximo de $15 \mathrm{mmHg}$. (Tabla 2).

Tabla 3. Frecuencias estadísticas en Protocolo anestésico 2 (Meloxicam, Diazepam y Ketamina).

\begin{tabular}{|c|c|c|c|c|c|}
\hline & & $\begin{array}{l}\text { Mediciones } \\
\text { basales } \\
\text { Grupo I }\end{array}$ & $\begin{array}{l}\text { Mediciones Grupo I } \\
\text { protocolo anestésico } 2 \\
\text { (min 5) }\end{array}$ & $\begin{array}{l}\text { Mediciones Grupo I } \\
\text { protocolo anestésico } \\
2(\text { min } 10)\end{array}$ & $\begin{array}{l}\text { Mediciones Grupo I } \\
\text { protocolo anestésico } \\
2 \text { (min } 15)\end{array}$ \\
\hline Media & & 20,40 & 21,60 & 9,44 & 11,75 \\
\hline Mediana & & 20,60 & 20,55 & 7,05 & 10,95 \\
\hline Moda & & $11,1^{*}$ & $4,7^{*}$ & $6,2^{*}$ & 14,7 \\
\hline Desviación Estándar & & 6,33 & 9,80 & 4,72 & 3,84 \\
\hline Varianza & & 40,19 & 96,04 & 22,33 & 14,81 \\
\hline Asimetría & & $-0,04$ & 0,003 & 0,84 & 0,31 \\
\hline $\begin{array}{l}\text { Error estándar de } \\
\text { asimetría }\end{array}$ & & 0,63 & 0,63 & 0,63 & 0,63 \\
\hline Curtosis & & $-1,28$ & $-0,88$ & $-0,68$ & $-0,84$ \\
\hline $\begin{array}{l}\text { Error estándar de } \\
\text { curtosis }\end{array}$ & & 1,23 & 1,23 & 1,23 & 1,23 \\
\hline Mínimo & & 11,1 & 4,7 & 3,7 & 6,6 \\
\hline Máximo & & 30,5 & 36,7 & 18,2 & 18,9 \\
\hline \multirow[t]{3}{*}{ Percentiles } & 25 & 14,62 & 13,52 & 6,20 & 8,77 \\
\hline & 50 & 20,60 & 20,50 & 7,05 & 10,95 \\
\hline & 75 & 25,87 & 31,90 & 13,87 & 14,77 \\
\hline
\end{tabular}

$\left(^{*}\right)$ Se muestra valor más pequeño 
De igual manera en la Tabla 3, los datos obtenidos suman un total de 12, al considerar las mediciones de ambos ojos en el Grupo I integrado por 6 conejos. En este caso, la media de las medidas basales es de $20,408 \mathrm{mmHg}$ y se incrementa al min 5 del Protocolo anestésico 2, luego disminuye sin llegar a valores más elevados que la basal. Al utilizar el protocolo anestésico 2 (Meloxicam, Diazepam y Ketamina), el valor de PIO más elevado fue de 36,7 $\mathrm{mmHg}$ al min 5, mientras que el mínimo valor es de 18,2 $\mathrm{mmHg}$ al min 10. En cuanto a la desviación estándar y varianza encontrados, se aprecian valores muy elevados como la varianza al min 5 con 96,045 por lo tanto, se considera una variabilidad de datos muy grande y una dispersión considerable.

En cuanto a la forma de distribución de los valores de $\mathrm{PIO}$, se tiene que las medidas basales poseen una asimetría negativa, es decir una curva con cola hacia la izquierda con valores menores a su media y en el caso de los valores de PIO durante anestesia, se trata de asimetrías positivas con cola hacia a derecha, es decir, valores mayores a sus medias. Sin embargo, al tratarse de datos muy variables y dispersos, se habla de una curtosis pequeña con valores de PIO alejados de la media, no homogénea. Tanto el error estándar se asimetría como el de curtosis, ratifican que no se podría tratar de una distribución normal, debido a variabilidad encontrada en la asimetría y curtosis.

Los valores mínimos, máximos y los percentiles, son descritos a continuación con ayuda de gráficos Box-plot, instrumento útil en caso de distribuciones asimétricas como sucede en este caso.

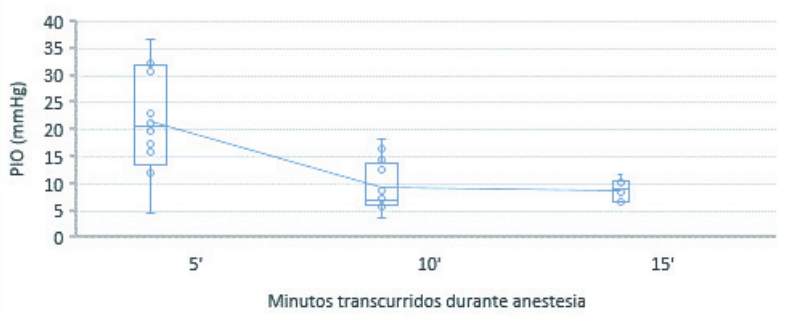

Figura 2. Distribución estadística de PIO - Protocolo anestésico 2 (Meloxicam, Diazepam y Ketamina).

Estas figuras 1 y 2 solo toman en cuenta las medidas de PIO obtenidas durante anestesia del Protocolo 2 (Meloxicam, Diazepam y Ketamina). Se observa la disminución en las medias 21,600 a 9,442 mmHg, valor que empieza a elevarse conforme pasa el tiempo hacia el min 15 con $11,758 \mathrm{mmHg}$, haciendo referencia a que el efecto del anestésico va perdiendo efecto. No se observan valores atípicos en ninguno de los minutos referenciales, haciendo ver estandarización de las mediciones. $\mathrm{Al}$ min 5, los valores máximos y mínimos se aprecian muy distantes ya que existe una mayor variabilidad de datos, pero estos mismos valores se van acercando al min 10 y 15 puesto que la variabilidad de datos se reduce al tener mediciones más homogéneas.

El cuartil 1 (Q1) denota que el 25\% de los valores de PIO están por debajo $13,52 \mathrm{mmHg}$, considerándose una PIO baja; mientras que el cuartil 3 (Q3), que el 75\% de los valores de PIO están por encima de 31,9 mmHg, PIO elevada. Los valores de PIO al min 10 de anestesia disminuyen y al finalizar al min 15 se aprecia menos variabilidad y dispersión de valores, incluso el valor mínimo de 6,6 se encuentra muy cercano al cuartil 1 (Q1) con $8,77 \mathrm{mmHg}$ a diferencia de los otros minutos referenciales con mayor lejanía entre estos últimos.

\section{DISCUSIÓN}

En el estudio realizado por Holve et al. (2013), el protocolo anestésico utilizado fue de ketamina con xilacina vía intramuscular (IM) para un grupo de conejos y la misma conjunción de fármacos vía intravenosa (IV) para otro grupo de conejos. Los valores resultantes vía IV representaron una disminución de la PIO desde el min 10 con $17,43 \mathrm{mmHg}$ hasta $15,60 \mathrm{mmHg}$ al min 25. De manera similar, en la vía IM, los valores de PIO desde el min 10 con $16,15 \mathrm{mmHg}$ hasta $15,08 \mathrm{mmHg}$ al min 2530; considerando las medidas basales de $20,15 \mathrm{mmHg}$ IV y de 19,03 mmHg IM.

En el presente estudio, se administraron los protocolos anestésicos 1 y 2 solo por vía intramuscular (Burke \& Potter, 1986; Brunton et al., 2013). En el grupo A de conejos, en donde se utilizó el Protocolo anestésico 1(Meloxicam, Xilacina y Ketamina), se obtuvieron valores de PIO en disminución desde $9,017 \mathrm{mmHg}$ al min 10 y de $8,350 \mathrm{mmHg}$ al min 15 . Mientras que en el caso del Grupo I de conejos utilizando el Protocolo anestésico 2 (Meloxicam, Diazepam y Ketamina), el valor de PIO obtenido al min 10 fue de $9,44 \mathrm{mmHg}$ y al min 15 , de $11,76 \mathrm{mmHg}$. Se observa que, en ambos casos, la PIO disminuye considerablemente al min 10 de anestesia, teniendo en cuenta los basales de ambos grupos con 19,92 y $20,41 \mathrm{mmHg}$ respectivamente. Por lo tanto, se puede decir que los fármacos utilizados en los protocolos 1 y 2 , actúan efectivamente disminuyendo los valores de PIO y que, con solo $10 \mathrm{~min}$ de haber transcurrido las anestesias, se apreció una diferencia grande teniendo como punto de partida los valores basales de PIO. 
Chandorkar et al. (1975), hizo uso de ketamina únicamente para observar las variaciones de la PIO. En este caso, las mediciones de PIO se realizaron por cada minuto transcurrido y las dosis utilizadas de ketamina fueron de 2,4 y $8 \mathrm{mg} / \mathrm{kg}$. Los resultados arrojaron una baja de la PIO de entre 2 y $8 \mathrm{mmHg}$ durante los min 7 y 8 , la caída de PIO continuó al usar $4 \mathrm{mg} / \mathrm{kg}$ de ketamina entre 8 y $16 \mathrm{mmHg}$ hasta el min 12 , ya no se observó ningún cambio significativo en cuanto a depresión de la PIO al usar $8 \mathrm{mg} / \mathrm{kg}$ de ketamina. Sin embargo, para la realización del presente estudio no se han encontrado otras investigaciones en conejos que avalen esta disminución de la PIO con el uso único de ketamina.

El presente estudio se realizó con protocolos anestésicos en donde se incluye a la ketamina como fármacos inductorio y pre-anestésicos que disminuyen la PIO. El protocolo anestésico 1 , fue de ketamina a dosis de 25 $\mathrm{mg} / \mathrm{kg}$ en el caso de su uso con xilacina y de $20 \mathrm{mg} / \mathrm{kg}$ al usarla junto con diazepam. Los resultados en el protocolo 1 muestra una disminución de $\mathrm{PIO}$ de $6 \mathrm{mmHg}$ del min 5 al 10, mientras que en el protocolo 2, una baja de hasta $17 \mathrm{mmHg}$, en los mismos min referenciales; disminución de PIO que se extendió hasta el min 15 en ambos casos. Cabe resaltar que en el estudio realizado por Chandorkar et al. (1975), las dosis de ketamina fueron mínimas a comparación del presente estudio, en donde se hizo uso de dosis actualizadas establecidas por la UC (2016).

Además, la vía de administración en el estudio por Chandorkar et al. (1975) fue intravenosa con un efecto más rápido de la ketamina, pero por menos tiempo; en contraposición con este estudio, en donde fue vía intramuscular teniendo un efecto más lento de acción, pero por más tiempo sumado al uso de fármacos preanestésicos, los cuales junto con la ketamina generan un plano anestésico más estable y manejable para la PIO en los conejos.

Ghaffari \& Moghaddassi (2010), obtuvieron las conjunciones de anestésicos descritas en el titulo vía intramuscular, y son de incrementos en la PIO durante el plano anestésico de hasta $35 \pm 4$ y $23 \pm 6 \mathrm{mmHg}$ para cada grupo de conejos respectivamente, tomando en cuenta los basales de entre 15 y $20 \mathrm{mmHg}$ para ambos grupos. La conjunción de ketamina-diazepam con dosis de $30 \mathrm{mg} / \mathrm{kg}$ y $1 \mathrm{mg} / \mathrm{kg}$ respectivamente resultaron en un aumento desde $23 \pm 8$ al min 5 a $35+-4$ al min 20 , mientras que en el presente trabajo se utilizó a dosis de $2 \mathrm{mg} / \mathrm{kg}$ de diazepam (IM) como pre-anestésico y $20 \mathrm{mg} / \mathrm{kg}$ de ketamina (IM) como inductorio, resultando en un ligero aumento al min 5 de $21,6 \mathrm{mmHg}$ desde una PIO base de $20,408 \mathrm{mmHg}$ pero, luego disminuye drásticamente al min 10 con $9,442 \mathrm{mmHg}$ y con $11,8 \mathrm{mmHg}$ al min 15 , manteniéndose disminuido respecto a la medición de PIO basal.

Es importante considerar que los fármacos administrados en combinación actúan de manera distinta, a diferencia de aquellos administrados por separado, tal y como se realizó en el presente estudio separando pre-anestésicos del fármaco inductorio. Otro factor a tomar en cuenta es que la dosis de ketamina utilizada por Ghaffari \& Moghaddassi (2010), fue $30 \mathrm{mg} / \mathrm{kg}$, es decir, $10 \mathrm{mg} / \mathrm{kg}$ más que la utilizada en el presente estudio con $20 \mathrm{mg} / \mathrm{kg}$ y en el caso de diazepam, sucede lo contrario, utilizan una dosis de $1 \mathrm{mg} / \mathrm{kg}$, mientras que en este estudio la dosis de diazepam es mayor con $2 \mathrm{mg} / \mathrm{kg}$. El diazepam posee la propiedad de disminuir la PIO y al ser utilizada en menor cantidad no se estaría obteniendo esa propiedad deseada, sobre todo cuando la dosis de ketamina es tan elevada, por tales motivos el estudio de Ghaffari \& Moghaddassi (2010) obtuvo mediciones de PIO elevadas, en vez de disminuciones consecutivas como en el presente estudio.

Es de suma importancia reconocer el uso del principio de las 3 Rs (reemplazo, reducción y refinamiento) en Bienestar Animal determinadas por la Asociación de Investigación Animal Europea, las cuales se utilizaron en el presente estudio; sin embargo, pudieron hacerse mejorías en el procedimiento al momento de la toma de medidas de PIO para evitar las situaciones de estrés, que tanto afectan a los lagomorfos por ser animales de presa (Pintor, 2005; Calasans-Maia et al., 2009; Cook \& Peiffer, 2010; Medrano-Palafox et al., 2010).

Se puede decir que las dosis establecidas de cada fármaco incluido en el protocolo anestésico 1, resultaron precisas para los propósitos del presente estudio, ya que se mantuvo una PIO reducida durante los 15 min de duración de mediciones (Duncalf, 1975; McLaren et al., 1996).

Podría establecerse un reajuste a la dosis de diazepam en el protocolo anestésico 2, con el fin de generar una disminución de PIO más certera, además establecer medidas necesarias para reducir al mínimo las posibles situaciones de estrés, las cuales generaron anomalías en la PIO en el presente estudio (Soto-Cabrera, 2010).

Debido a la variabilidad y dispersión de datos encontrada en las frecuencias estadísticas tanto del protocolo anestésico 1 como en el 2, sería importante considerar un tamaño muestral mayor con el fin de obtener datos con menor variabilidad, mayor homogeneidad y una 
consecuente reducción de errores. Resulta importante considerar la PIO al momento de formular protocolos anestésicos en conejos, ya que un alza considerable en PIO podrías significar la ocurrencia de un daño ocular o la exacerbación de alguna patología ocular previa a anestesia. Los resultados de este estudio significan un aporte a considerar al momento de establecer protocolos anestésicos en conejos y representan datos importantes para la formulación de nuevos trabajos de investigación en la misma área de estudio (Baños-Díez \& March-Pujol, 2002; Calasans-Maia et al., 2009; AVEPA, 2014; Alzate, 2015).

\section{REFERENCIAS BIBLIOGRÁFICAS}

Alzate, M. A. 2015. Técnicas de anestesia para cirugia oftálmica. Monografía Estudiante. Universidad de Antioquía. Recuperado de http://bibliotecadigital.udea.edu.co/ bitstream/10495/3839/1/AlzateaAna_2016_ TecnicasAnesteciaCirugia.pdf

AVEPA(Asociación de Veterinarios Españoles Especialistas en Pequeños Animales). 2014. Actualización en anestesia y analgesia. Recuperado de http:// avepa.org/pdf/proceedings/ANESTESIA_ PROCEEDINGS2014.pdf

Baños-Díez, J.E. \& March-Pujol, M. 2002. Farmacología ocular. Barcelona, España. Ed. UPC. Recuperado de https://books.google.com.pe/books?id=H9w W_9Zu9HIC\&pg=PA189\&lpg=PA189\&dq=e fectos+de+aine+sistemico+en+presion+intraocu lar\&source $=$ bl $\&$ ots $=$ rf $45 X 5 Y 1 \mathrm{ek} \& \operatorname{sig}=1 \mathrm{rYY7X}$ usNtJaD_j6s7okjsD5qHw\&hl=es\&sa=X\&ved $=2 \mathrm{ahUKEwjn} 6 \mathrm{bm} 5 \mathrm{tJvfAhUj} 11 \mathrm{kKHeABBicQ6}$ AEwBnoECAUQAQ\#v=onepage\&q=ef

Brunton, L.L.; Lazo, J.S. \& Parker, K.L. 2013. Gooman \& Gilman's The Pharmacologicalbasis of Therapeutics. In: Brunton, L.L. (Ed.) (1 $11^{\text {th }}$ Ed.). California: McGraw-hill. Recuperado de https://dvmbooks. weebly.com/uploads/2/2/3/6/22365786/2. goodman_and_gilman.pdf

Burke, J.A. \& Potter, D.E. 1986. The ocular effects of xylazine in rabbits, cats, and monkeys. Journal of Ocular Pharmacology and Therapeutics, 2: 9-21.
Calasans-Maia, M.D.; Monteiro, M.L.; Áscoli, F.O. \& Granjeiro, J.M. 2009. The rabbit as an animal model for experimental surgery. Acta Cirurgica Brasileira, 24: 325-328.

Castellvi, J.; Parera, M. \& Loscos, J. 2009. Consideraciones sobre los principios físicos de la tonometría de aplanación. Gaceta Óptica, 442: 30-34.

Chandorkar, A.G.; Jain, P.K. \& Albal, M.V. 1975. Modulations in intraocular pressure under ketamine anaesthesia. Indian Journal of Ophthalmology, 23: 22-24.

Cook, C.S. \& Peiffer, R.L. 2010. Conocimientos clínicos básicos. pp. 1-12. Peiffer, R. \& Petersen-Jones, S. (Eds). Oftalmología de pequeños animales. Ed. Harcourt SA, España. Recuperado de http://www.veterinaria.org/descargas/libros/ oftalmologia2.pdf

Duncalf, D. 1975. Anesthesia and intraocular pressure. Bulletin of the New York Academy of Medicine, 51: 374-381.

Ghaffari, M.S. \& Moghaddassi, A.P. 2010. Effects of ketamine-diazepam and ketamine-acepromazine combinations on intraocular pressure in rabbits. Veterinary Anaesthesia and Analgesia, 37: 269-272. https://doi.org/10.1111/J.14672995.2010.00531.X

Hedenqvist, P. 2005. Anaesthesia and analgesia for surgery in rabbits and rats: $A$ comparison of the effects of different compounds. Recuperado de https:// www.metris.nl/media/documents/laboras/ Publications/2008 - Hedenqvist - Anaesthesia and Analgesia in rats compound comparison. pdf

Holve, D.L.; Gum, G.G. \& Pritt, S.L. 2013. Effect of sedation with xylazine and ketamine on intraocular pressure in New Zealand white rabbits. Journal of the American Association for Laboratory Animal Science, 52: 488-490.

Kern, T. J. 1997. Rabbit and rodent ophthalmology. Seminars in Avian and Exotic Pet Medicine, 6: 138-145.

McLaren, J.W.; Brubaker, R.F. \& Fitzsimon, J.S. 1996. Continuous measurement of intraocular 
pressure in rabbits by telemetry. Investigative Ophthalmology and Visual Science, 37: 966975.

Medrano-Palafox, J.; Martín Baiza-Durán, L.; Angélica, D.; Contreras-Rubio, Y.; Álvarez-Delgado, J.; Kira Chávez-Villa, D. \& Urquides-Espinoza, G. 2010. Presión intraocular en conejos albinos Nueva Zelanda: experiencia con tonómetro de Goldman, Revista Mexicana de Oftalmología, 84: $1-4$.

Pietro-Calvo, E. 2012. Farmacocinética, biodisponibilidad y tolerancia ocular de memantina en conejos: Aportaciones al análisis de una nueva formulación oftálmica a base de nanopartículas. Universidad de Zaragoza. Recuperado de https://zaguan. unizar.es/record/9904/files/TESIS-2012-138. pdf

Pintor, J. 2005. Nuevas perspectivas farmacológicas de la melatonina en el tratamiento de las patologías oculares. Anales de La Real Academia Nacional de Farmacia, 71, 429-438.

Raw, D. \& Mostafa, S. 2001. Drugs and the eye. British Journal of Anaesthesia and continuous medical education and professional development Reviews, 1: 161-165.
Snyder, K.C.; Lewin, A.C., Mans, C. \& McLellan, G. J. 2018. Tonometer validation and intraocular pressure reference values in the normal chinchilla (Chinchilla lanigera). Veterinary Ophthalmology, 21: 4-9.

Soto-Cabrera, M.A. 2010. Descripción del comportamiento anestésico del conejo doméstico (oryctolagus cuniculus) frente a la inducción y redosificación con ketamina intravenosa (iv). Universidad de Chile. Recuperado de http://repositorio.uchile.cl/ bitstream/handle/2250/131375/Descripcióndel-comportamiento-anestesico-del-conejodomestico-\%28 Oryctolagus-cuniculus $\% 29$ frente-a-la-induccion-y-redosificacioncon-ketamina-intravenosa-\%28IV\%29. pdf?sequence $=1$

UC (University of Colorado). 2016. Veterinary Anesthetic and Analgesic Formulary. University of Colorado. Denver. Anschutz Medical Campus. $3^{\text {er }}$ Ed. Version G.

Zamira, M.; Rodríguez, T. \& Ayala, E.E. 2014. Cunicultura. Recuperado de http://ri.uaemex. $\mathrm{mx} / \mathrm{bitstream} / \mathrm{handle} / 20.500 .11799 / 31394 /$ secme-19206.pdf?sequence $=1$

Received October 15, 2019.

Accepted November 27, 2019. 